\title{
Case report: five-year experience of AKT inhibition with miransertib (MK-7075) in an individual with Proteus syndrome
}

\author{
Christopher A. Ours, Julie C. Sapp, Mia B. Hodges, Allison J. de Moya, \\ and Leslie G. Biesecker \\ Center for Precision Health Research, National Human Genome Research Institute, National Institutes \\ of Health, Bethesda, Maryland 20892, USA
}

\begin{abstract}
Proteus syndrome is a rare overgrowth disorder caused by postzygotic activating variants in AKT1. Individuals may develop a range of skin, bone, and soft tissue overgrowth leading to functional impairment and disfigurement. Therapy for this disorder is limited to supportive care and surgical intervention. Inhibitors of AKT, originally designed as cancer therapeutics, are a rational, targeted pharmacologic strategy to mitigate the devastating morbidity of Proteus syndrome. We present the 5-yr follow-up of an 18-yr-old male with Proteus syndrome treated with miransertib (MK-7075), an oral pan-AKT inhibitor. At completion of a planned 48-wk phase 1 pharmacodynamic study, the individual derived sufficient benefit that the study was amended to permit continued use and assess the long-term safety of miransertib. The treatment has been well-tolerated with mild treatment-attributed side effects including headache, transient hyperglycemia, and transient elevations of aspartate aminotransferase, alanine aminotransferase, and bilirubin. The patient has experienced sustained improvement of pain and slowed growth of bilateral plantar cerebriform connective tissue nevi. This case report supplements the data from our prior study extending those findings out to 5 years. It shows that at the doses used, miransertib has a favorable safety profile and durable benefit of improving symptoms of pain and slowing progression of overgrowth in Proteus syndrome in a single individual. Although an uncontrolled single report cannot prove safety or efficacy, these data lend support to the encouraging preliminary data of our prior phase 1 pharmacodynamic study.
\end{abstract}

Corresponding author: chris.ours@nih.gov

(C) 2021 Ours et al. This is a work of the US Government.

Ontology terms: bone pain; connective tissue nevi; multiple skeletal anomalies; overgrowth

Published by Cold Spring Harbor Laboratory Press

doi:10.1101/mcs.a006134

\section{INTRODUCTION}

Proteus syndrome is a rare mosaic overgrowth disorder that causes a wide range of signs and symptoms. Proteus syndrome most frequently manifests as overgrowth of the skin, bone, and soft tissue in the first few years of life. The hallmark lesion is the cerebriform connective tissue nevus (CCTN), which typically affects the plantar surface of the foot (Beachkofsky et al. 2010). Other features of Proteus syndrome include vascular malformations, organomegaly, cystic lung disease, an increased risk of thrombosis, and tumor predisposition. Manifestations of Proteus syndrome vary among individuals in both anatomic distribution and severity. Although phenotypically heterogeneous, the disorder is unified by a mosaicactivating variant in AKT1, most commonly c.49G > A, p.(Glu17Lys), and relentless progression of disease, particularly in childhood (Lindhurst et al. 2011). 
The PI3K/AKT signaling pathway plays a critical role in the regulation of cell proliferation, growth, and survival and is often dysregulated in cancer (Hoxhaj and Manning, 2020). Precision oncology efforts to inhibit this pathway have led to the U.S. Food and Drug Administration (FDA) approval of PI3K inhibitors idelalisib and copanlisib for subtypes of relapsed lymphoma and alpelisib, a PI3Ka-specific inhibitor, for PIK3CA-mutated breast cancer (Miller et al. 2015; Narayan et al. 2021). Whereas malignancies often harbor multiple genomic or epigenomic alterations leading to neoplastic behavior, Proteus syndrome is caused by a single somatic variant. This makes it an attractive opportunity for repurposing inhibitors of the PI3K/AKT pathway developed for oncology.

There are important differences in the approach to the treatment of malignancy and overgrowth syndromes to consider. Inhibition of AKT1 in Proteus syndrome is likely to require a prolonged, perhaps lifelong, duration, as compared to the shorter duration used to induce apoptosis in malignancy (Song et al. 2019). By analogy, methotrexate is used at high intermittent intravenous doses in the treatment of acute lymphoblastic leukemia but is prescribed at lower continuous oral doses for an extended time in the treatment of rheumatoid arthritis (Weinblatt 2013; Larsen et al. 2016). In the study of overgrowth disorder therapy, there is a lesser tolerance for acute toxicity and increased focus of potential chronic toxicity. A narrower therapeutic window may also influence decisions of dosing, resulting in lower dosing for the treatment of overgrowth disorders. The objective of therapy as either a primary treatment or primary prevention may further influence dosing requirements.

Miransertib (MK-7075, formerly ARQ 092) is an orally administered allosteric pan-AKT inhibitor that has been studied in the oncology setting as well as in Proteus syndrome and related overgrowth disorders (Yu et al. 2015; Forde et al. 2021). From experience in oncology trials, dose-limiting toxicities of miransertib were hyperglycemia and skin rash at $80 \mathrm{mg}$ daily (Saleh et al. 2013). These side effects are common among inhibitors of the PI3K/AKT pathway (Hyman et al. 2017; Juric et al. 2018). Previous reports of miransertib use in Proteus syndrome at doses ranging from $10 \mathrm{mg}$ daily to $100 \mathrm{mg}$ daily suggest a tolerable side effect profile and reduction in the progression of Proteus syndrome. One report describes tolerance of miransertib at $25 \mathrm{mg} / \mathrm{m}^{2}$ (50 mg per dose) daily over one year in an individual who experienced only mild side effects including dry mouth and gingivostomatitis. Stagnation of overgrowth of the skull, abdominal wall lipomatosis, and decreased CCTN growth were observed (Biesecker et al. 2020). Another individual with Proteus syndrome developed a low-grade ovarian carcinoma and achieved a partial response of the tumor with miransertib at $100 \mathrm{mg}$ daily for $7 \mathrm{~d}$ on/7 d off every $2 \mathrm{wk}$. She was treated for $22 \mathrm{mo}$ at the time of the report. Side effects included skin rash, nausea, hyperglycemia, and elevation of aspartate aminotransferase (AST) and alanine aminotransferase (ALT), which were mild to moderate and transient. Additionally, she experienced qualitative improvement in physical function and quality of life (Leoni et al. 2019).

A phase 1 pharmacodynamic study of miransertib enrolled six individuals with Proteus syndrome. This trial was a $3+3$ dose escalation study that met its primary end point, a $50 \%$ reduction in AKT phosphorylation in affected skin, at the starting oral dose of $5 \mathrm{mg} /$ $\mathrm{m}^{2}$ daily. A single individual in this study discontinued miransertib because of elevations in liver function enzymes and $\gamma$-glutamyl transferase (GGT). The laboratory abnormalities resolved after discontinuation of miransertib. Other side effects in this cohort were mild and included fatigue, headache, mouth sores, and nausea. This study also reported mitigation of overgrowth and improvement in pain among the three pediatric participants (KepplerNoreuil et al. 2019).

We present the 5-yr follow-up of an individual with Proteus syndrome who was initially enrolled on the phase 1 pharmacodynamic study and elected to continue treatment with miransertib. 


\begin{tabular}{|c|c|c|c|c|c|c|c|}
\hline Gene & Chromosome & $\begin{array}{l}\text { HGVS DNA } \\
\text { reference }\end{array}$ & $\begin{array}{l}\text { HGVS protein } \\
\text { reference }\end{array}$ & $\begin{array}{l}\text { Variant } \\
\text { type }\end{array}$ & Predicted effect & $\begin{array}{c}\mathrm{dbSNP} / \mathrm{db} \text { ar } \\
\text { ID }\end{array}$ & Genotype \\
\hline AKT1 & Chr 14 & NM_005163.2 & NP_005154.2 & Substitution & $\begin{array}{c}\text { c. } 49 \mathrm{G}>\mathrm{A}, \\
\text { p.(Glu17Lys) }\end{array}$ & rs121434592 & Mosaic \\
\hline
\end{tabular}

\section{RESULTS}

\section{Clinical Presentation}

The individual is an 18-yr-old male who has participated in our Natural History of Proteus Syndrome Study (Clinicaltrials.gov NCT00001403) since he was 6 years old and enrolled in the pharmacodynamic study when he was 13 years old. When he enrolled on the phase 1 study (Clinicaltrials.gov NCT02594215), he had a history of leg length discrepancy requiring multiple surgeries, bilateral plantar CCTN, musculoskeletal pain of the lower back and lower extremities, eczema, seasonal allergies, iron deficiency anemia, and irritable bowel syndrome. The mosaic variant in AKT1 c.49G > A, p.(Glu17Lys) was present in cultured fibroblasts from an affected skin biopsy (Table 1).

Following completion of the originally planned 48 wk of miransertib as part of the phase 1 study, the individual expressed a desire to continue miransertib because of improvement in pain and slowing of CCTN growth. The protocol was amended to provide continued use of miransertib with the aim of evaluating longer-term safety and durability of response (i.e., continued decrease in pain and slowing of CCTN growth). There was a 9 mo pause prior to resuming miransertib at $5 \mathrm{mg} / \mathrm{m}^{2}$ once daily. Based on the demonstration of safety at dosing of $15 \mathrm{mg} / \mathrm{m}^{2}$ and $25 \mathrm{mg} / \mathrm{m}^{2}$ daily from an industry-sponsored expanded access program and a perceived decrease of effect, the dose was increased to $10 \mathrm{mg} / \mathrm{m}^{2}$ daily $16 \mathrm{wk}$ after resuming miransertib. The magnitude of dose escalation, doubling the daily drug exposure, was selected to minimize potential of adverse events and was based on the dose level from the original $3+3$ study design of the phase 1 trial. He remains on $10 \mathrm{mg} / \mathrm{m}^{2}$ (20 mg per dose) daily at the time of this report.

\section{Safety Analyses}

Overall, miransertib has been well-tolerated. During the individual's treatment period there have been no toxicity-related prescribed discontinuations. Concomitant medications have included occasional use of over-the-counter analgesics (acetaminophen and ibuprofen) and medications for seasonal allergies (cetirizine and fluticasone nasal spray). Interval electrocardiogram (ECG) and echocardiograms have been normal except for a persistent sinus bradycardia, which was present at time of initiating miransertib. Weight and height centiles at miransertib initiation were 44th centile and 96th centile, respectively. Weight and height centiles 5 years later were 81 st centile and 99th centile, respectively.

Since initiating miransertib, he has experienced a total of 33 adverse events (AEs) summarized in Table 2. The most common AEs were upper respiratory infection, pharyngitis, and dry skin. Thirty-one (94\%) of the AEs were either grade 1 or grade 2 events. There have been two grade $3 \mathrm{AEs}$, each determined to be unrelated to miransertib. Three years after starting miransertib, he sustained a tibial plateau fracture and anterior cruciate ligament sports-related injury that required hospitalization and surgical repair. He continued to take miransertib after the orthopedic surgery and did not experience any surgical complications or delayed healing. The second grade 3 event he experienced was a pharyngeal infection with phlegmon requiring a brief hospitalization for intravenous antibiotics. He 


\begin{tabular}{|c|c|c|c|c|}
\hline Adverse event & Grade 1 & Grade 2 & Grade 3 & Total \\
\hline \multicolumn{5}{|l|}{ Cardiac disorders } \\
\hline Sinus bradycardia & 1 & & & 1 \\
\hline \multicolumn{5}{|l|}{ Gastrointestinal disorders } \\
\hline Abdominal pain & 1 & & & 1 \\
\hline Nausea & & 1 & & 1 \\
\hline Vomiting & & 1 & & 1 \\
\hline Dry mouth & 2 & & & 2 \\
\hline \multicolumn{5}{|l|}{ General } \\
\hline Fatigue & 1 & & & 1 \\
\hline Pain & 1 & 1 & & 2 \\
\hline \multicolumn{5}{|l|}{ Infections } \\
\hline Upper respiratory infection & 1 & 4 & & \\
\hline Pharyngitis & 3 & & 1 & 4 \\
\hline \multicolumn{5}{|l|}{ Injury, poisoning, and procedural complications } \\
\hline Fracture, tibia and anterior cruciate ligament tear & & & 1 & 1 \\
\hline Fracture, calcaneus & & 1 & & 1 \\
\hline \multicolumn{5}{|l|}{ Investigations } \\
\hline Aspartate aminotransferase increased ${ }^{a}$ & 1 & & & 1 \\
\hline Alanine aminotransferase increased ${ }^{a}$ & 1 & & & 1 \\
\hline Blood bilirubin increased $^{a}$ & 3 & & & \\
\hline \multicolumn{5}{|l|}{ Metabolism disorders } \\
\hline Hyperglycemia $^{a}$ & 2 & & & 2 \\
\hline Increased triglycerides ${ }^{a}$ & 1 & & & 1 \\
\hline \multicolumn{5}{|l|}{ Nervous system disorders } \\
\hline Headache $^{a}$ & 1 & & & 1 \\
\hline \multicolumn{5}{|l|}{ Respiratory disorders } \\
\hline Allergic rhinitis & & 1 & & 1 \\
\hline \multicolumn{5}{|l|}{ Skin disorders } \\
\hline Dry skin & 3 & & & 3 \\
\hline Total & 22 & 9 & 2 & 33 \\
\hline
\end{tabular}

aPossibly or probably related to miransertib.

recovered without surgical intervention or drainage. The individual also underwent epiphysiodesis to permanently arrest growth of the left leg. This was not included in the summary of adverse events as it is an anticipated intervention for bone overgrowth in Proteus syndrome.

During the treatment period, he had two separate sports-related fractures, a tibial fracture and later calcaneal fracture. Vitamin D, 25-Hydroxy level $(33 \mathrm{ng} / \mathrm{mL})$, and bone mineral density by dual energy $X$-ray absorptiometry (Z-score 1.0) were obtained 6 mo after calcaneal fracture, which was $5 \mathrm{yr}$ from miransertib initiation. These did not show clinically significant vitamin $\mathrm{D}$ deficiency or osteoporosis.

Adverse events determined to be possibly or probably related to miransertib included transient elevations of AST ( $\max 38 \mathrm{U} / \mathrm{L})$, ALT ( $\max 32 \mathrm{U} / \mathrm{L}$ ), total bilirubin (max $1.3 \mathrm{mg}$ / $\mathrm{dL}$ ), and direct bilirubin $(0.6 \mathrm{mg} / \mathrm{dL})$, hyperglycemia $(\max 117 \mathrm{mg} / \mathrm{dL})$, increased triglycerides 
(max $164 \mathrm{mg} / \mathrm{dL}$ ), and headache. These were all grade 1 AEs and spontaneously resolved within 12-16 wk of identification without dose adjustment.

\section{Treatment Effect and Durability Decreased Rate of CCTN Growth}

The individual has bilateral plantar CCTN. The left foot is more severely affected than the right. Prior to starting miransertib, the left CCTN involved 39.2\% of the left plantar surface and the right CCTN involved $9.5 \%$ of the right plantar surface. Following 48 wk of treatment, the left CCTN involved $40.3 \%$ of the left plantar surface and right CCTN involved $10.5 \%$ of the right plantar surface. Five years after initiating miransertib, the left CCTN involves $44.6 \%$ of the left plantar surface and the right CCTN involves $15.2 \%$ of the right plantar surface (Fig. 1).

The annual increase in percent of the plantar surface involved by CCTN during the 7 years prior to treatment with miransertib was $5.3 \%$ and $4.8 \%$ on the left and right, respectively. The annual increase in percent of the plantar surface involved by CCTN during the 5 years after initiating miransertib was $1.2 \%$ and $1.2 \%$ on the left and right, respectively (Fig. 2).

\section{Improvement in Patient-Reported Outcomes}

There were four patient-reported outcome measures used in this study; three included a parent proxy form (Fig. 3). The NRS-11 scale was used to report the most severe pain in the preceding $7 \mathrm{~d}$ of an evaluation. This score at baseline was 7 out of 10, at $48 \mathrm{wk}$ was 3 , and $5 \mathrm{yr}$ after initiation was 0 . At the time of the aforementioned calcaneal fracture the score increased to 5. The Pain Interference Index (PII) score, which ranges from 0 to 6, decreased over time by self-report and remained constant by proxy report. The baseline mean PII scores were 0.67 (self) and 0 (proxy). After 5 years, his mean Pll scores were 0 (self) and 0 (proxy). The physical function PROMIS upper extremity forms showed no impairment at baseline and after 5 years. This was an expected finding in this individual who does not have upper extremity overgrowth. The physical function PROMIS mobility forms showed minimal to no impairment in self-report, but proxy-report T-score increased during the treatment period indicating improved function. The baseline T-score was 39 and after 5 years increased to 56 . The Impact of Pediatric Illness (IPI) score increased over time indicating an improved quality of life. The baseline IPI score was 93 (self) and 90 (proxy) and after 5 years was 97 (self) and 96 (proxy).

\section{Genomic Analysis}

Mosaic c.49G > A, p.(Glu17Lys) was identified by Sanger sequencing and confirmed by restriction fragment length polymorphism assay of DNA from cultured fibroblasts from affected skin. This finding has been previously reported as part of a larger cohort (Lindhurst et al. 2011).

\section{DISCUSSION}

These observations support previous reports describing tolerability of lower-dose (compared to oncology dosing) miransertib in Proteus syndrome and provides evidence of the long-term safety of miransertib in childhood. The continued decrease in pain and CCTN growth is consistent with our preliminary evidence of benefit in the pharmacodynamic study (Keppler-Noreuil et al. 2019) and importantly provides preliminary evidence that miransertib may be durably effective at improving symptoms and mitigating overgrowth in Proteus syndrome. Prior natural history data in this and other patients has shown an average annual 


\section{A Left Foot}

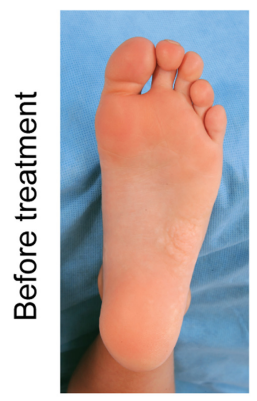

6

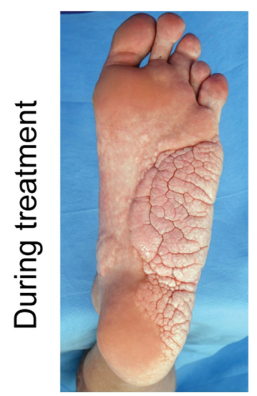

13

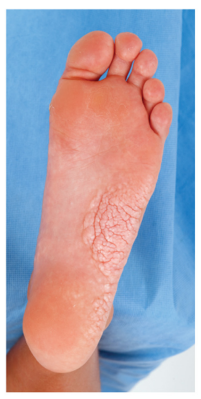

8.5

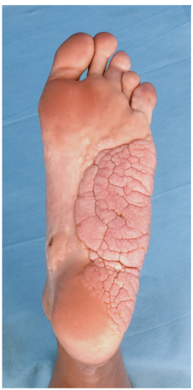

14

B Right Foot

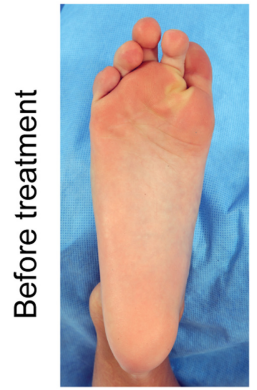

6

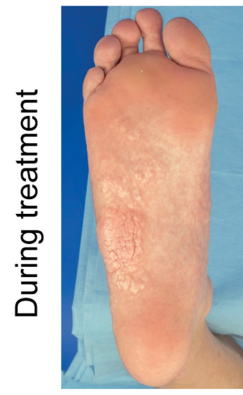

13

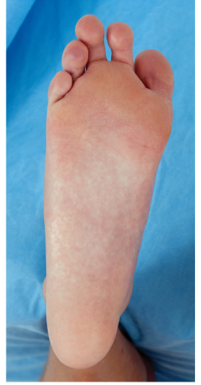

8.5

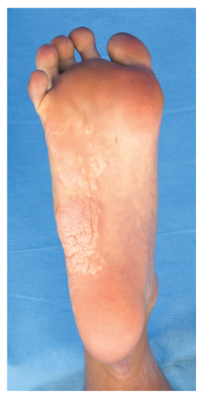

14

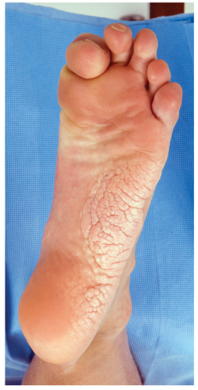

9.6

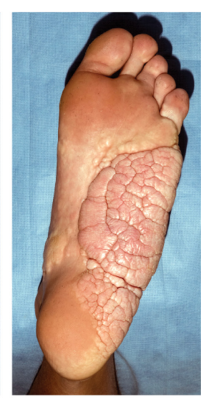

14.8

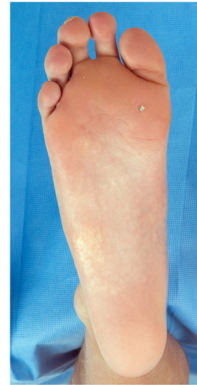

9.6

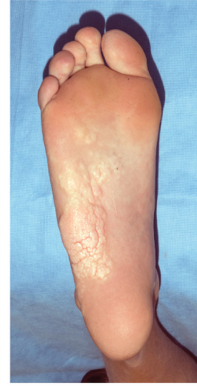

14.8

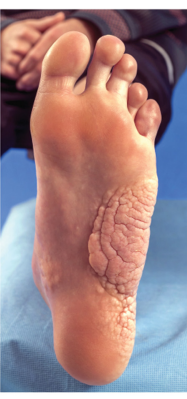

10.6

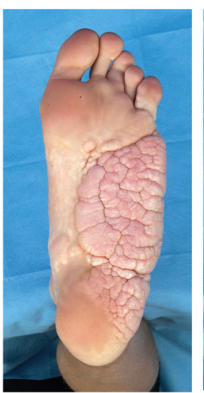

16

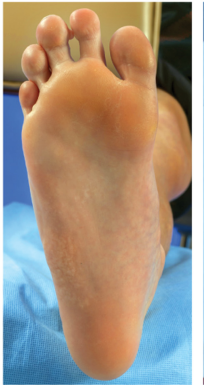

10.6

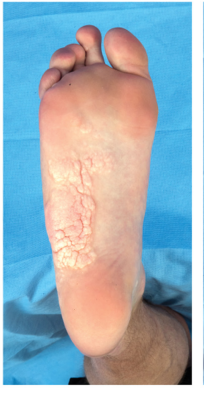

16

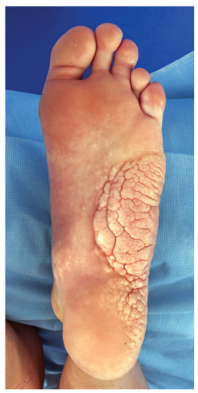

11

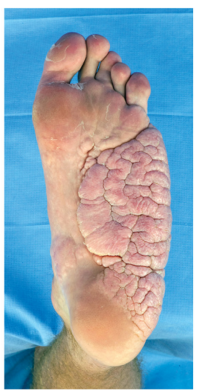

17.6

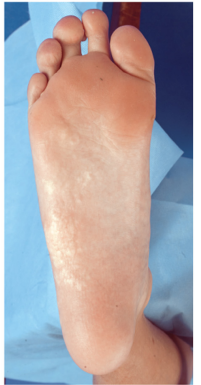

11

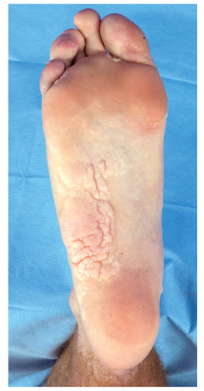

17.6

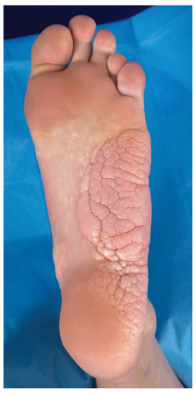

12

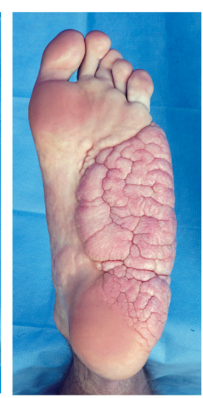

18

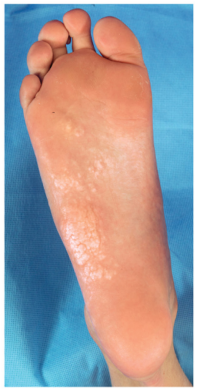

12

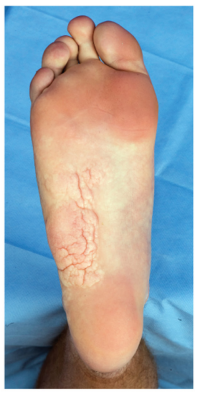

18

Figure 1. Serial photography of the left $(A)$ and right $(B)$ feet showing the progression of the cerebriform connective tissue nevus (CCTN) occurring more rapidly before initiating miransertib and a slowing of the involvement of the plantar surface during miransertib treatment. 


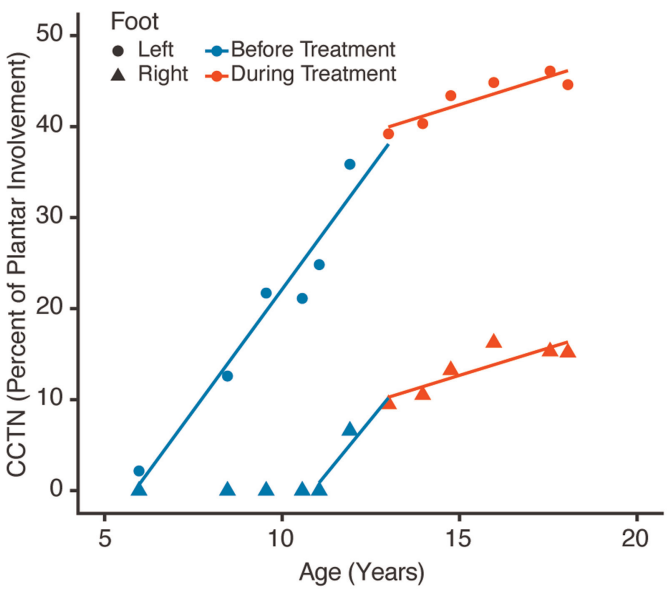

Figure 2. Measurement of the percent of cerebriform connective tissue nevus (CCTN) involvement on the plantar surface. The CCTN development and growth were observed prior to miransertib. A decrease in the annual growth rate is observed following initiation of miransertib and was sustained during the treatment period.

growth of 5.6\% (Nathan et al. 2018). In contrast to therapeutic approaches in oncology that aim to eliminate malignant cells often by providing maximally tolerated doses over a limited duration, treatment of mosaic overgrowth disorders seeks to dampen overactivity of molecular signaling over long periods of time, possibly lifelong. This necessitates that treatment not only be efficacious but tolerable for many years and, in the treatment of children, not interfere with normal patterns of growth and development.

The optimal dosing for mosaic overgrowth disorders is likely to be less than that used in oncology. Miransertib has been well-tolerated in this individual at a dose of $10 \mathrm{mg} / \mathrm{m}^{2}(20$ $\mathrm{mg}$ daily). This dose contrasts with early phase oncology trials in adults that recommended phase 2 doses ranging from $60 \mathrm{mg}$ daily to $200 \mathrm{mg}$ daily every other week (Saleh et al. 2013; Harb et al. 2015). At lower doses of miransertib, skin rashes and hyperglycemia have been mild in individuals with Proteus. However, early discontinuation of treatment in an individual on the phase 1 pharmacodynamic study because of elevation in liver enzymes signals the importance of laboratory monitoring. The individual described here has had mild increases in total and direct bilirubin, ALT, AST, and fasting glucose. These increases were transient and did not require intervention or change in miransertib dosing.

Treatment with miransertib did not interfere with normal patterns of growth and development. His height centiles remained nearly constant (96th-99th centile) during the treatment period, and he had normal pubertal development. The increase in weight from the 44th to 81 st centile coincided with pubertal growth and improved his body mass index from less than 3 rd centile at study entry to 25 th centile at most recent evaluation. Another important consideration in a population that often requires surgical intervention is the effect of miransertib on wound healing (Li et al. 2016). Two years after starting miransertib, he underwent percutaneous epiphysiodesis with transphyseal screws of the left distal femur and proximal tibia to arrest overgrowth of the left leg. He had no postoperative complications or delayed wound healing following this procedure nor after an anterior cruciate ligament repair. Per previous guidance regarding the increased risk of deep vein thrombosis in Proteus syndrome, he was prescribed perioperative prophylactic anticoagulation for these procedures (Crenshaw et al. 2018).

The effect of miransertib on features of Proteus syndrome was evaluated in this individual through photography and patient-reported outcome measures. The individual's primary 

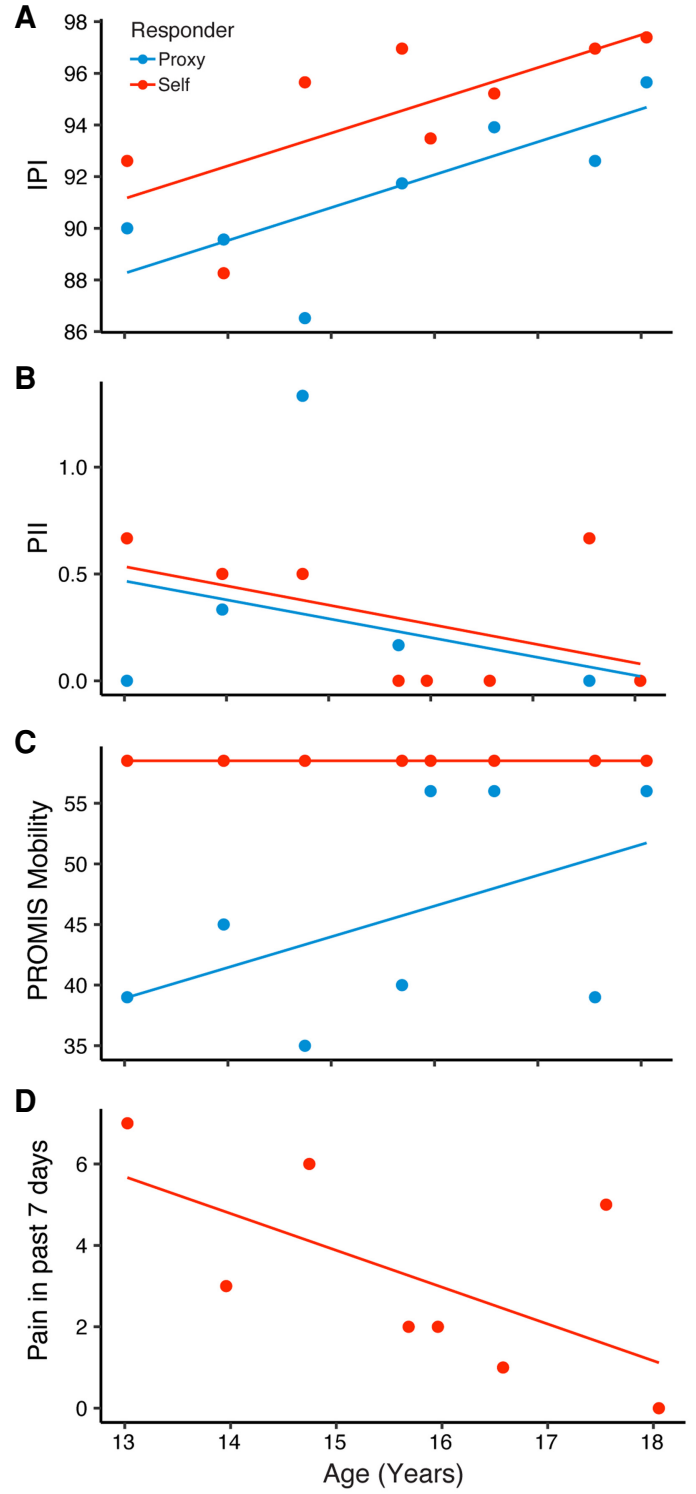

Figure 3. Patient-reported outcome measures improved during the treatment period. (A) Impact of Pediatric Illness (IPI), (B) Pain Interference Index (PII), (C) PROMIS Mobility, and (D) NRS-11 scale of most severe pain in past $7 \mathrm{~d}$.

manifestations of Proteus syndrome were lower extremity overgrowth and CCTN. His quality of life was most affected by a decrease in pain of the lower back and legs, which was a primary motivation to continue miransertib. The rate of growth of the left and right CCTN decreased while on treatment. Prior to starting miransertib, the increase in CCTN involvement of the plantar surface was similar to the previously reported natural history rate of $5.6 \%$ per year (Nathan et al. 2018). However, during the 5 years since starting miransertib, annual growth decreased to $1.2 \%$ per year. Whereas the previously reported natural history rate was modeled as a linear rate of growth during ages $\leq 18 \mathrm{yr}$ and a lower linear rate after 18 $y r$, it is possible that this rate may actually slow gradually as individuals approach adulthood and may have contributed to the decreased CCTN growth observed in this individual. 
Patient reported outcomes of quality of life, physical function, and pain improved during the 5 -yr period. There are limitations to the use of these measures such as the 5-yr-long duration of repeated measures as well as a potential ceiling effect of the IPI in this individual with baseline scores of 90 and above.

This case represents the longest reported use of miransertib in Proteus syndrome. This experience in a single individual supports the long-term safety of miransertib, but larger studies are needed. The effect of miransertib on other manifestations of Proteus syndrome, such as cystic lung disease and progressive scoliosis, was not studied in this report. Prospective trials that include the range of the Proteus syndrome phenotype are required to assess other potential benefits of miransertib in Proteus syndrome. Further investigation of the efficacy and long-term safety of miransertib are planned in a phase 2 clinical trial (Clinicaltrials.gov NCT04316546).

\section{METHODS}

\section{Safety}

Safety evaluations including physical examination, laboratory investigations, and ECG occurred every 12 wk with interval assessment by telephone every 4 wk. ECGs were obtained annually. The Common Terminology Criteria for Adverse Events version 4.0 (CTCAEv4.0) was used to categorize toxicity. Determinations of relatedness to miransertib were made by the investigators.

\section{Measurement of CCTN}

Photography of bilateral plantar CCTN was assessed as previously described (Nathan et al. 2018). There were six pairs (left and right feet) of photographs before initiating miransertib beginning at age $6 \mathrm{yr}$, and six pairs of photographs afterward ending at age $18 \mathrm{yr}$. Measurements of plantar area (excluding toes) and CCTN were performed independently by two individuals (M.B.H. and A.J.D.). The percent of CCTN involvement was calculated as the ratio of CCTN area to plantar area. The annual rate of change in percent of plantar involvement of CCTN was estimated using linear regression. Volumetric magnetic resonance images of the ankles and feet were obtained to further characterize the size of the CCTN and bone overgrowth. However, these were challenging to interpret because of difficulties demarcating the lesions and high interobserver variance (Keppler-Noreuil et al. 2019).

\section{Patient-Reported Outcomes}

Patient-reported outcome measures included the NRS-11 pain scale, IPI quality of life scale, PROMIS physical function upper extremity short form 8a, PROMIS physical function mobility short form 8a, and the PII (Dworkin et al. 2005; Martin et al. 2015; Schalet et al. 2016). The NRS-11 pain scale was used to ask the individual's most severe pain in the past $7 \mathrm{~d}$ on a scale of 0 to 10 , with 0 representing "no pain" and 10 the "worst pain you can imagine." The IPI is a 43-item questionnaire with ordinal responses on a five-point scale (1-5; "not at all" to "a lot"). Raw scores were calculated by summation of responses valued 1 through 5 , with reversal of negative items and scaled to a score of 1-100. A higher score indicates better quality of life (Martin et al. 2015). The PROMIS physical functioning self-report and proxy forms for upper extremity and mobility each have eight items. The raw score, derived from the sum of values of the responses to each question, is converted to a T-score to standardize the result. A higher T-score indicates high levels of physical function. Reference data for the general population of the United States have mean T-score of 50 with standard deviation of 10. The Pll scale is a six-item questionnaire with responses from 0 to 6 . The score is the mean 
Competing Interest Statement L.G.B. is a member of the lllumina Corp. Medical Ethics Board, has received in-kind research support from ArQule, Inc. (now wholly owned by Merck, Inc.), and receives honoraria from Cold Spring Harbor Laboratory Press.

Received July 27, 2021; accepted in revised form October 1, 2021. of the total of the items for a maximal score of 6. A lower score is desired indicating a decrease in pain's interference with daily activities.

Forms appropriate for age at enrollment were completed by the participant throughout the treatment period. Proxy versions of the IPI, PROMIS, and PII scales were completed the participant's mother. These are presented descriptively without statistical analysis.

\section{ADDITIONAL INFORMATION}

\section{Data Deposition and Access}

The genetic results have been previously deposited to COSMIC (https://cancer.sanger.ac .uk/cosmic) under accession number COSP31880 as part of a previous publication (Lindhurst et al. 2011).

\section{Ethics Statement}

We obtained written informed consent from the participant's parent and assent from the participant. Written informed consent of the participant was obtained when he reached age of majority. The National Institutes of Health Institutional Review Board has approved both the Study of Proteus Syndrome and Related Congenital Disorders (Clinicaltrials.gov NCT00001403) and Dose Finding Trial of MK-7075 in Children and Adults with Proteus Syndrome (Clinicaltrials.gov NCT02594215).

\section{Acknowledgments}

The authors thank the individual and his family for their long-standing participation in this research. We also thank Julia Fekecs for aid in the preparation of figures.

\section{Author Contributions}

C.A.O., J.C.S., and L.G.B. conceived and designed this work. All authors contributed to data collection. C.A.O. and L.G.B. performed data analysis and interpretation. C.A.O. drafted the manuscript, and all authors provided critical review and approval of the final version.

\section{Funding}

Miransertib was provided by Merck. Funding was provided by the National Human Genome Research Institute Intramural Research Program, HG200388-07.

\section{REFERENCES}

Beachkofsky TM, Sapp JC, Biesecker LG, Darling TN. 2010. Progressive overgrowth of the cerebriform connective tissue nevus in patients with Proteus syndrome. J Am Acad Dermatol 63: 799-804. doi:10.1016/ j.jaad.2009.12.012

Biesecker LG, Edwards M, O'Donnell S, Doherty P, MacDougall T, Tith K, Kazakin J, Schwartz B. 2020. Clinical report: one year of treatment of Proteus syndrome with miransertib (ARQ 092). Cold Spring Harb Mol Case Stud 6: a004549. doi:10.1101/mcs.a004549

Crenshaw MM, Goerlich CG, Ivey LE, Sapp JC, Keppler-Noreuil KM, Scott AC, Biesecker LG, Tosi LL. 2018. Orthopaedic management of leg-length discrepancy in Proteus syndrome: a case series. J Pediatr Orthop 38: e138-e144. doi:10.1097/BPO.0000000000001121

Dworkin RH, Turk DC, Farrar JT, Haythornthwaite JA, Jensen MP, Katz NP, Kerns RD, Stucki G, Allen RR, Bellamy N, et al. 2005. Core outcome measures for chronic pain clinical trials: IMMPACT recommendations. Pain 113: 9-19. doi:10.1016/j.pain.2004.09.012

Forde K, Resta N, Ranieri C, Rea D, Kubassova O, Hinton M, Andrews KA, Semple R, Irvine AD, Dvorakova V. 2021. Clinical experience with the AKT1 inhibitor miransertib in two children with PIK3CA-related overgrowth syndrome. Orphanet J Rare Dis 16: 109. doi:10.1186/s13023-021-01745-0 
Harb W, Saleh M, Papadopoulos K, Chai F, Larmar M, Abbadessa G, Schwartz B, Tolcher A. 2015. Clinical trial results from the subgroup of lymphoma/CLL in a phase 1 study of ARQ 092, a novel pan AKT-inhibitor. Blood 126: 5116. doi:10.1182/blood.V126.23.5116.5116

Hoxhaj G, Manning BD. 2020. The PI3K-AKT network at the interface of oncogenic signalling and cancer metabolism. Nat Rev Cancer 20: 74-88. doi:10.1038/s41568-019-0216-7

Hyman DM, Smyth LM, Donoghue MTA, Westin SN, Bedard PL, Dean EJ, Bando H, El-Khoueiry AB, PérezFidalgo JA, Mita $\mathrm{A}$, et al. 2017. AKT inhibition in solid tumors with AKT1 mutations. J Clin Oncol 35: 2251-2259. doi:10.1200/JCO.2017.73.0143

Juric D, Rodon J, Tabernero J, Janku F, Burris HA, Schellens JHM, Middleton MR, Berlin J, Schuler M, GilMartin M, et al. 2018. Phosphatidylinositol 3-kinase a-selective inhibition with alpelisib (BYL719) in PIK3CA-altered solid tumors: results from the first-in-human study. J Clin Oncol 36: 1291-1299. doi:10 1200/JCO.2017.72.7107

Keppler-Noreuil KM, Sapp JC, Lindhurst MJ, Darling TN, Burton-Akright J, Bagheri M, Dombi E, Gruber A, Jarosinski PF, Martin S, et al. 2019. Pharmacodynamic study of miransertib in individuals with Proteus syndrome. Am J Hum Genet 104: 484-491. doi:10.1016/j.ajhg.2019.01.015

Larsen EC, Devidas M, Chen S, Salzer WL, Raetz EA, Loh ML, Mattano LA, Cole C, Eicher A, Haugan M, et al. 2016. Dexamethasone and high-dose methotrexate improve outcome for children and young adults with high-risk B-acute lymphoblastic leukemia: a report from children's oncology group study AALL0232. J Clin Oncol 34: 2380-2388. doi:10.1200/JCO.2015.62.4544

Leoni C, Gullo G, Resta N, Fagotti A, Onesimo R, Schwartz B, Kazakin J, Abbadessa G, Crown J, Collins CD, et al. 2019. First evidence of a therapeutic effect of miransertib in a teenager with Proteus syndrome and ovarian carcinoma. Am J Med Genet A 179: 1319-1324. doi:10.1002/ajmg.a.61160

Li G, LiYY, Sun JE, Lin WH, Zhou RX. 2016. ILK-PI3K/AKT pathway participates in cutaneous wound contraction by regulating fibroblast migration and differentiation to myofibroblast. Lab Invest 96: 741-751. doi:10 .1038/labinvest.2016.48

Lindhurst MJ, Sapp JC, Teer JK, Johnston JJ, Finn EM, Peters K, Turner J, Cannons JL, Bick D, Blakemore L, et al. 2011. A mosaic activating mutation in AKT1 associated with the Proteus syndrome. N Engl J Med 365: 611-619. doi:10.1056/NEJMoa1104017

Martin S, Nelson Schmitt S, Wolters PL, Abel B, Toledo-Tamula MA, Baldwin A, Wicksell RK, Merchant M, Widemann B. 2015. Development and validation of the English Pain Interference Index and pain interference index-parent report. Pain Med 16: 367-373. doi:10.1111/pme.12620

Miller BW, Przepiorka D, de Claro RA, Lee K, Nie L, Simpson N, Gudi R, Saber H, Shord S, Bullock J, et al. 2015. FDA approval: idelalisib monotherapy for the treatment of patients with follicular lymphoma and small lymphocytic lymphoma. Clin Cancer Res 21: 1525-1529. doi:10.1158/1078-0432.CCR-14-2522

Narayan P, Prowell TM, Gao JJ, Fernandes LL, Li E, Jiang X, Qiu J, Fan J, Song P, Yu J, et al. 2021. FDA approval summary: alpelisib plus fulvestrant for patients with HR-positive, HER2-negative, PIK3CA-mutated, advanced or metastatic breast cancer. Clin Cancer Res 27: 1842-1849. doi:10.1158/1078-0432.CCR-203652

Nathan NR, Patel R, Crenshaw MM, Lindhurst MJ, Olsen C, Biesecker LG, Keppler-Noreuil KM, Darling TN. 2018. Pathogenetic insights from quantification of the cerebriform connective tissue nevus in Proteus syndrome. J Am Acad Dermatol 78: 725-732. doi:10.1016/j.jaad.2017.10.018

Saleh M, Papadopoulos K, Arabnia A, Patnaik A, Stein RM, Cattaneo F, Abbadessa G, Greenberg J, Warren S, Tolcher A. 2013. Abstract LB-197: first-in-human study with ARQ 092, a novel pan AKT-inhibitor: results from the advanced solid tumors cohorts. Cancer Res 73. doi:10.1158/1538-7445.AM2013-LB-197

Schalet BD, Hays RD, Jensen SE, Beaumont JL, Fries JF, Cella D. 2016. Validity of PROMIS physical function measured in diverse clinical samples. J Clin Epidemiol 73: 112-118. doi:10.1016/j.jclinepi.2015.08.039

Song M, Bode AM, Dong Z, Lee MH. 2019. AKT as a therapeutic target for cancer. Cancer Res 79: 1019-1031. doi:10.1158/0008-5472.CAN-18-2738

Weinblatt ME. 2013. Methotrexate in rheumatoid arthritis: a quarter century of development. Trans Am Clin Climatol Assoc 124: 16-25.

Yu Y, Savage RE, Eathiraj S, Meade J, Wick MJ, Hall T, Abbadessa G, Schwartz B. 2015. Targeting AKT1-E17K and the PI3K/AKT pathway with an allosteric AKT inhibitor, ARQ 092. PLoS ONE 10: e0140479. doi:10 .1371 /journal.pone.0140479 


\section{COLD SPRING HARBOR Molecular Case Studies}

\section{Case report: five-year experience of AKT inhibition with miransertib (MK-7075) in an individual with Proteus syndrome}

Christopher A. Ours, Julie C. Sapp, Mia B. Hodges, et al.

Cold Spring Harb Mol Case Stud 2021, 7: a006134 originally published online October 14, 2021 Access the most recent version at doi: $10.1101 /$ mcs.a006134

References This article cites 23 articles, 5 of which can be accessed free at: http://molecularcasestudies.cshlp.org/content/7/6/a006134.full.html\#ref-list-1

License This is a work of the US Government.

Email Alerting Receive free email alerts when new articles cite this article - sign up in the box at the Service top right corner of the article or click here. 